\title{
Some remarks on PM2.5
}

\author{
Marco Casazza and Angelo Piano \\ Dipartimento di Fisica Generale «A. Avogadro», Università di Torino, Italy
}

\begin{abstract}
Since 1970, the General Physics Department of «Università degli Studi di Torino» has carried out a project research, on inorganic solid particulate matter. The special issue of Annals of Geophysics, published for Professor Giorgio Fiocco's 70th birthday, gives us the possibility to make some important remarks on this topic, focusing on PM2.5. This has been possible using all the old and new experimental data of the measures made by the authors of this paper since 1970 .
\end{abstract}

Key words PM2.5 - solid inorganic particulate matter

\section{Introduction}

Since 1970, the General Physics Department of «Università degli Studi di Torino» has carried out a research project on inorganic solid Particulate Matter (PM) (Longhetto et al., 1975; Bacci et al., 1983). The purpose was to obtain not only knowledge about the mass concentration of PM2.5, but also to understand the correlations with climatological and meteorological factors. Recently, we devoted more attention to indoor measurements and on outdoor-indoor influences. The special issue of Annals of Geophysics, published for Professor Giorgio Fiocco's 70th birthday, gives us the possibility to make some important remarks on this topic. This has been possible collecting the experimental data of measures (old and new ones) made by the authors of this over during more than 30 years.

The particles, dispersed in the atmosphere, with an aerodynamic diameter of less than $2.5 \mu \mathrm{m}$ (PM2.5) have been studied with great accuracy

Mailing address: Dr. Angelo Piano, Dipartimento di Fisica Generale «Avogadro», Università di Torino, Via P. Giuria 1, Torino, Italy; e-mail: piano@to.infn.it because, being considered the respirable fraction of Suspended Particulate Matter (SPM), they can reach the inner parts of the respiratory apparatus and hence the alveoli.

\section{Dimensional spectra and associated chemical elements}

Around 1970 we started to measure the concentrations of inorganic particles, for the purpose of characterising the emissions of sources of great intensity and the immission in the environment in the neighbourhood of the studied sources. At that time, the granulometric distribution of the dimensions of particles, expressed as $\Delta N / \Delta$ (logr) (\% of total), was in good agreement with the theoretical one of Noll and Pilat (1971), for dust of continental origin.

We saw, analysing more than $2 \cdot 10^{6}$ samples collected both in urban and rural sites, using the technique of Atomic Absorption Spectrophotometry (AAS), that the chemical elements associated to the Suspended Particulate Matter (SPM), like $\mathrm{Pb}, \mathrm{Cd}, \mathrm{V}$, are associated with particles of smaller diameter (fig. 1), while $\mathrm{Al}, \mathrm{Cu}, \mathrm{Fe}$ are not so much linked to PM2.5 (fig. 2).

After 25 years, the granulometric distribution has changed and, today, particularly in the urban environment, a typical representation of its trend can be shown using fig. 3 . 


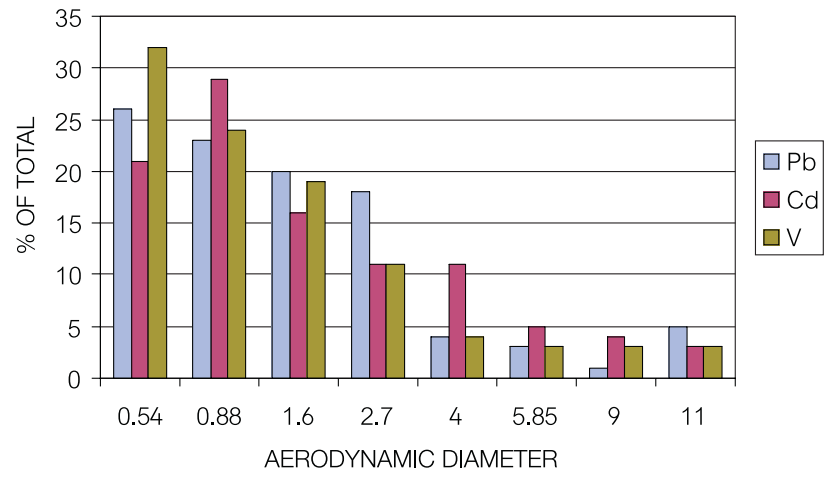

Fig. 1. Means of 256 measures of $\mathrm{Pb}, \mathrm{Cd}$ and $\mathrm{V}$ granulometric distributions in several different places in the Po Valley.

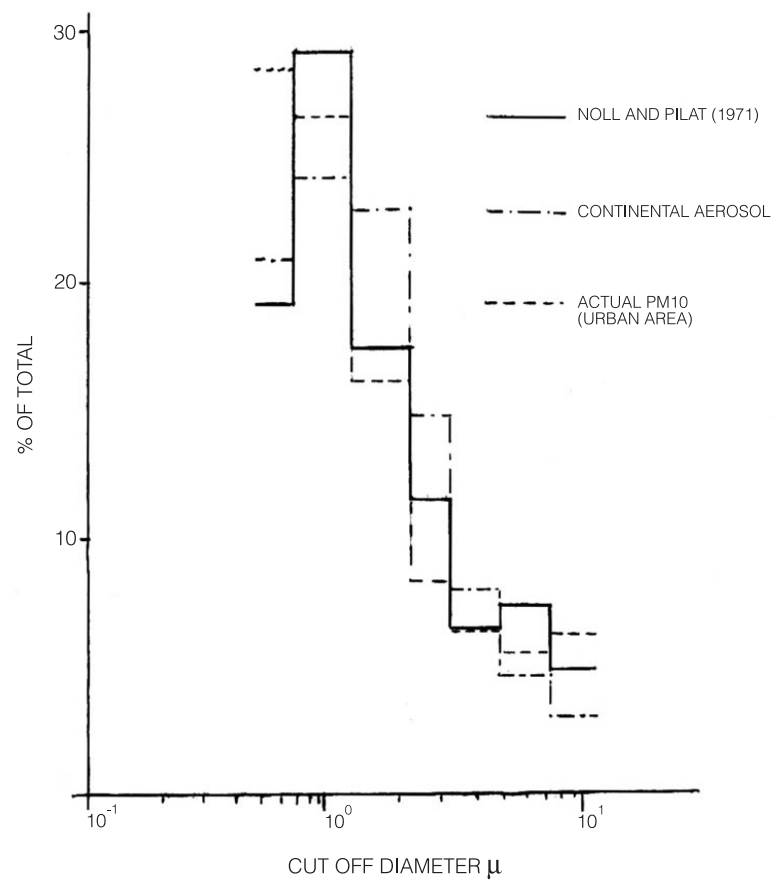

Fig. 3. Current and old (25 years ago) granulometric distributions of inorganic particulate matter with aerodynamic diameter from $0.43 \mu \mathrm{m}$ to $11 \mu \mathrm{m}$ compared with Noll and Pilat's (1971) theoretical continental granulometric aerosol distribution.

This distribution of mass concentration was obtained using an Andersen 2000 Inc. (1977) non-viable cascade impactor (Andersen, 1958; Vaughan, 1989), which is composed of 8 different stages and permits the collection of PM with aerodynamic diameter of less than $11 \mu \mathrm{m}$.

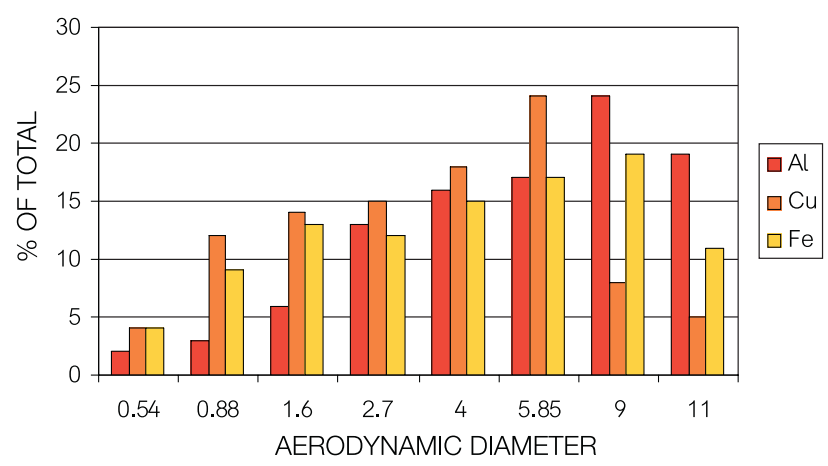

Fig. 2. Means of 384 measures of $\mathrm{Al}, \mathrm{Cu}$ and $\mathrm{Fe}$ granulometric distributions carried out in Northern Italy.

The fraction with aerodynamic diameter of less than $0.8 \mu \mathrm{m}$ is much increased respect to the particles of larger dimensions. This is confirmed by the growth by two orders of magnitude, of the concentration of number per unit volume, of Aitken nuclei (diameters from $0.01 \mu \mathrm{m}$ to $0.1 \mu \mathrm{m})$, that, nowadays, in urban environment, reach some units multiplied by $10^{5}$ per unit volume $\left(\mathrm{cm}^{3}\right)$.

\section{Correlation with meteorological factors}

We considered it useful to have continuous measures of mass concentration. Those were made using a DustTrak 8520 aerosol monitor, produced by TSI (2001). Many temporal sequences, during the last 6 years, and the knowledge of the contemporary measures of the values of the meteorological parameters, such as solar radiation, wind speed and direction, relative humidity, temperature and quantity of rain, give us the possibility to understand the influence of many phenomena on the mass concentration trend. The meteorological measures were carried out in several sites of Northern Italy and, in particular, in the Turin CNR area, where RASS, SODAR and other devices are present to measure vertical profiles of wind, relative humidity and temperature.

We can see, as an example, in fig. 4, a fragment of a temporal sequence collected during March 2001. 


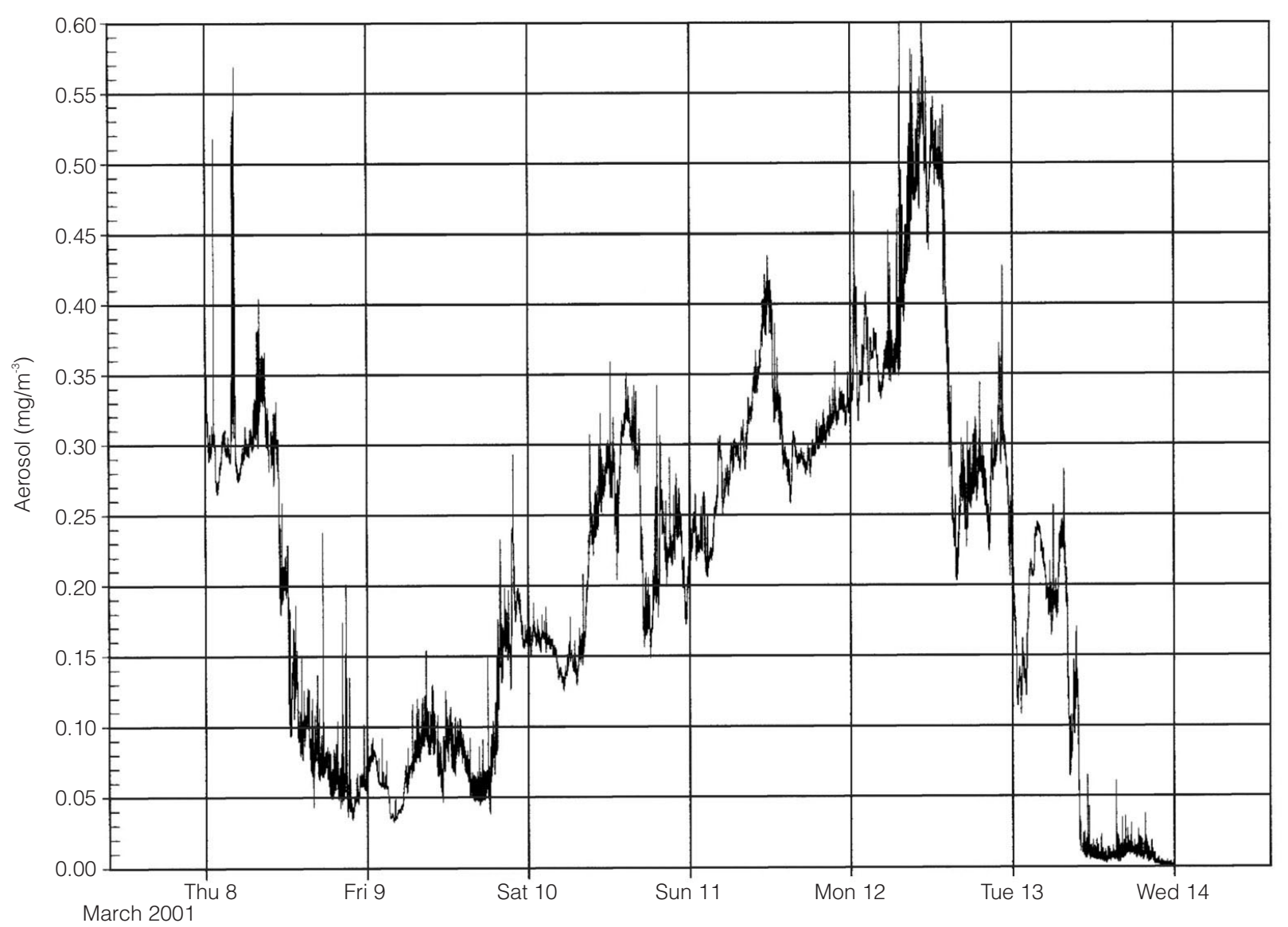

Fig. 4. Temporal sequence in urban area obtained using DustTrak 8520 in March 2001.

The data used for this graph are automatically collected, with different programmable sampling times, by the instrument and analysed by its software, that furnishes a graphic representation of the mass concentration trend, like the one shown above, with an error of $2 \mu \mathrm{g} \mathrm{m}^{-3}$. We showed this measure of 6 days because it starts and finishes with a rainfall and because it is visible how, in urban areas during winter, an accumulation phenomenon is present that can lead to aerosol concentrations higher than $0.5 \mathrm{mg} \mathrm{m}^{-3}$. This is evident from Friday 9th and Tuesday 13th (note that the increasing trend is present on Sunday too, when it can be referred only to road traffic or to domestic heating). The spikes that are present during the different days are linked with diurnal, meteorological or local factors.

Using some other figures, we will give an interpretation to some recurrent phenomena, mak- ing a qualitative comparison between PM2.5 and some meteorological variables.

Figure 5 shows the PM2.5 concentration trend compared with the variations of wind speed. In this case wind is responsible, moving air masses, of the visible spikes and of the dilution of the PM2.5 concentration in the examined area.

Figure 6 shows a period characterized by the presence of a rainfall. PM2.5, considering its dimensions, is not reduced directly and immediately by rain itself but, more easily, by its associated wind. A deeper analysis of this phenomenon becomes necessary considering that, in every rainfall event, a variable period of stable concentration is present, visible under the form of a more or less pronounced bend in the graph.

Figure 7 helps us to explain this particular concentration trend. After the beginning of the rainfall, after a fall in PM2.5 mass concentration, both relative humidity and PM2.5 show an 

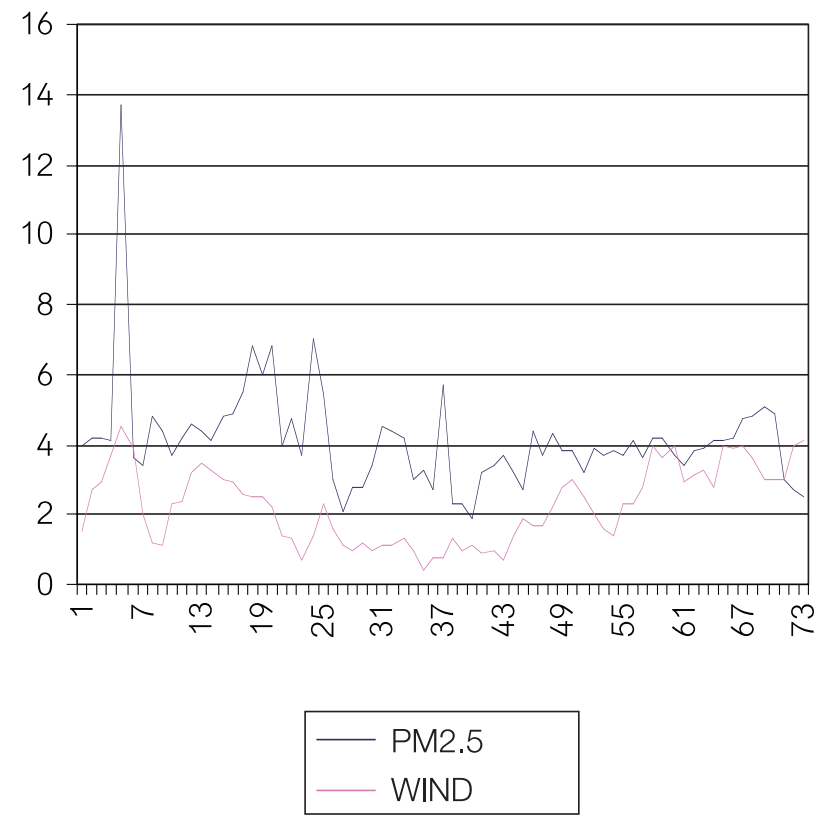

Fig. 5. Qualitative comparison between wind speed and PM2.5 mass concentration.

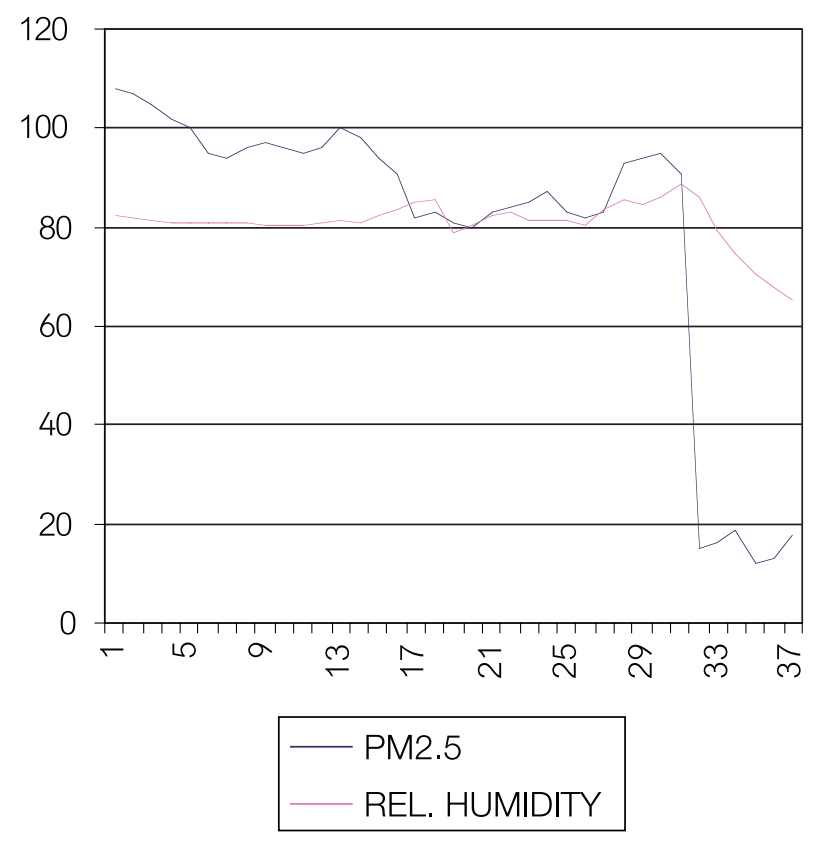

Fig. 7. Qualitative comparison between relative humidity and PM2.5 trends.

increase, while, after that, a further decrease of the two parameters considered is visible. This might be explained considering that the aerosol might be composed of condensation nuclei too,

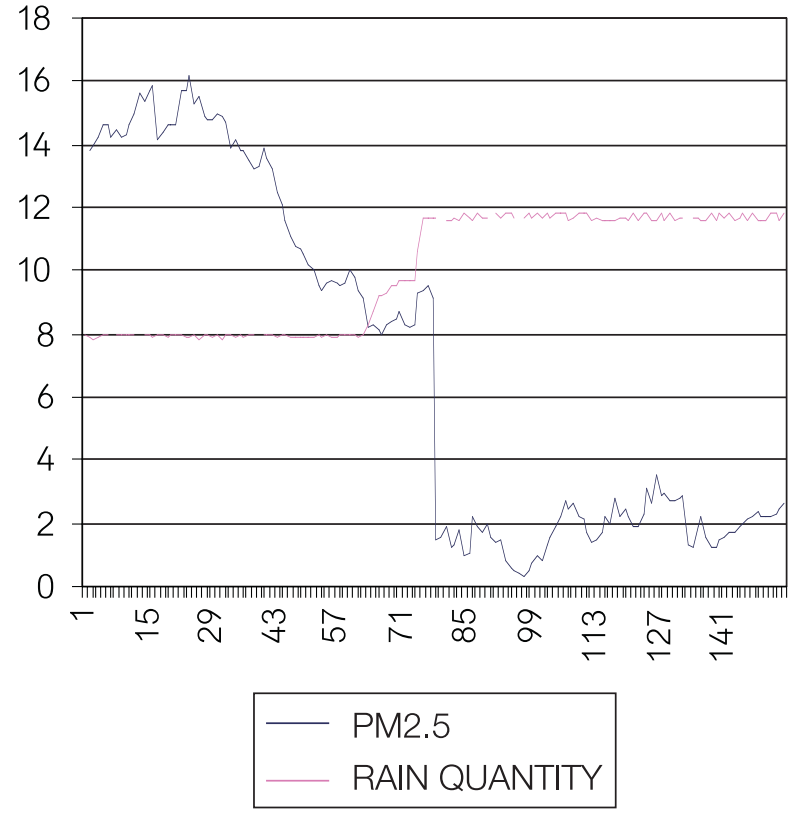

Fig. 6. Qualitative comparison between rain trend and PM2.5 concentration.

that can actively participate in the precipitation thereby making a contribution to the reduction of relative humidity.

Figure 8 shows the frequently found, in the case of very fine weather conditions, correlation between the PM2.5 concentration trend and the variation of the solar radiation during a day of measures.

Using different (more than $2.5 \cdot 10^{6}$ measures of 1 min each) of this kind and the related meteorological data, we can affirm that:

- PM2.5 are not immediately removed by rainfalls.

- They are taken away by wind or taken around the point of measure from areas with different rate of pollution.

- They have a diurnal modulation, linked with traffic and industrial production.

- They reflect particular local emissions.

- In conditions of dynamic equilibrium, they characterise the area in which the measures take place and the activities done in that particular place.

- They are a witness more of the path followed by different air masses than their peculiar characteristics. 

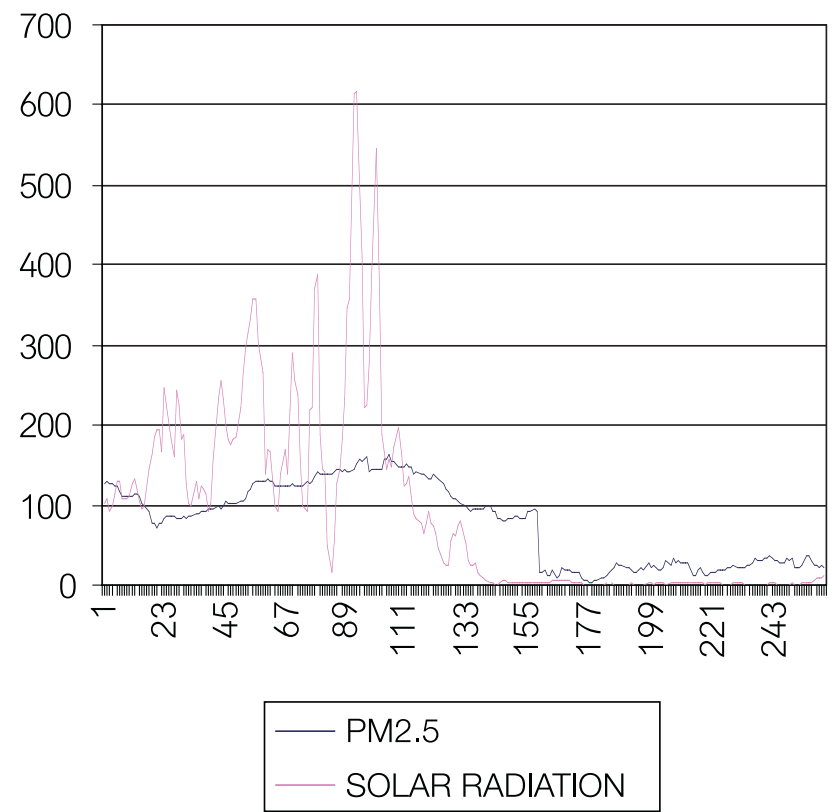

Fig. 8. Trends of PM2.5 and solar radiation.

\section{Indoor measures}

The last two characteristics found experimentally are of great interest for indoor measures, considering, in particular, environments without particular sources and without smokers, like an office.

If particular sources are present, like the smoke of one cigarette, as shown in fig. 9, it is possible to see a rapid and marked increase in PM2.5 concentration; the time needed to return to the original values of concentration can be some days. The measure presented here was taken in an office of about $70 \mathrm{~m}^{3}$ in conditions of thermal equilibrium.

In terms of Aitken nuclei, we can say that the inspired and expired smoke contains, in number concentration, on average, $7 \cdot 10^{5}$ particles $\mathrm{cm}^{-3}$.

The other characteristics, deduced observing the results of many (more than $10^{3}$ ) indoor mea-

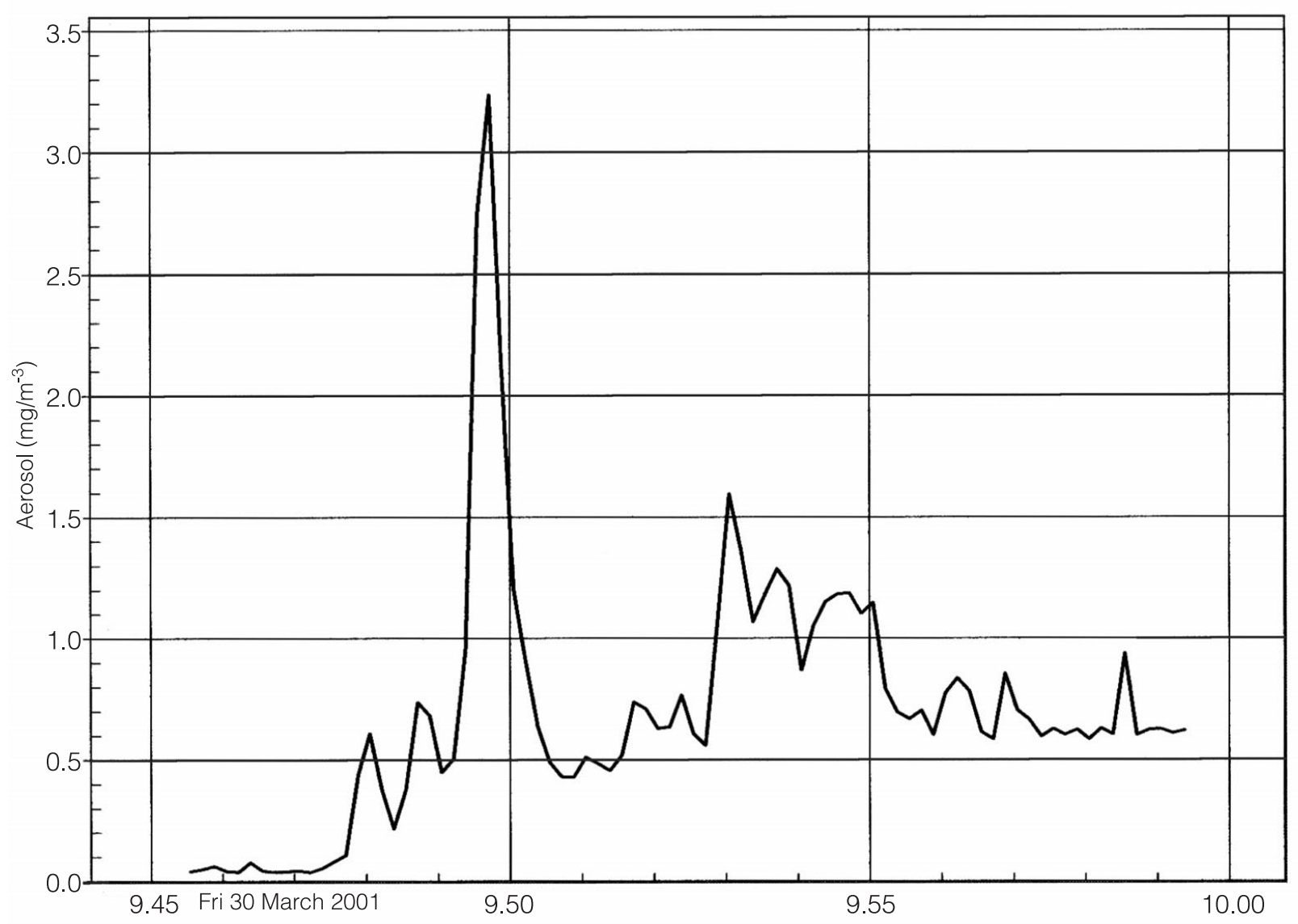

Fig. 9. PM2.5 mass concentration induced by cigarette smoke in $70 \mathrm{~m}^{3}$ room in thermal equilibrium. 
sures (domestic too), can be summarised saying that:

- Household appliances intaking air (vacuum cleaners and so on ...) do not retain, through the filter, PM2.5.

- Common domestic activities lift or generate PM2.5 (mainly during cleaning or cooking or frying).

- The exchange with outdoor air depends on pre existing concentrations (indoor and outdoor) and on differences of temperature.

- Often, the operation of changing the air of an indoor environment has the effect of inducing a worse air quality.

\section{Conclusions}

Using the data of our measures, we can affirm that PM2.5 concentrations are greatly increasing. We are studying the chemical proprieties of this fraction of SPM, including its mutagenesis to define its danger. PM2.5 concentrations are more poisonous indoors than outdoors, especially if there are some intense local sources, among which we can consider a smoker or some common household appliances.
A better knowledge on PM2.5 can lead to important interventions, useful to reduce the risks for human health.

\section{REFERENCES}

ANDERSEN, A.A. (1958): A new sampler for the collection, sizing and enumeration of viable particles, J. Bacteriol., 76, 471-484.

ANDERSEN 2000 INC. (1977): Operating manual for Andersen 2000 Inc. 1 ACFM ambient particle sizing samplers (Andersen 2000 Inc., Atlanta, Georgia).

Bacci, P., A. Longhetto, G. Marcazzan, A. Piano, F. Prodi, C. SABbioni and A. Ventura (1983): Aerosol characterization in a Po Valley site, in Proceedings of the Gesellschaft für Aerosolforschung meeting, J. Aerosol Sci., 14 (3), 222-225.

LONGHETTO, A., P. PAVESE and A. PiAnO (1975): Comparison among granulometric distributions of $\mathrm{Pb}$ and $\mathrm{Cd}$ on urban, bottom valley, and mountain atmospheric aerosols, in Proceedings of the 13th International Meeting on Alpine Meteorology, Riv. Ital. Geofis., 1, 95-96.

NolL, K.E. and M.J. PILAT (1971): Size distribution of atmospheric giant particles, Atmos. Environ., 5, 527540.

TSI (2001): Aerosol Monitor DustTrak 8520 Operating Instruction.

VAUGHAN, N.P. (1989): The Andersen impactor: calibration, wall losses and numerical simulation, J. Aerosol Sci., 20, 67-90. 